\title{
Realtime Control of Distributed Generation for Voltage Stability Improvement and HV Side Support
}

\author{
Khaled Alzaareer \\ Electrical Engineering Department \\ École de technologie supérieure \\ Montreal, Quebec, Canada \\ khaled.alzaareer.1@ems.etsmtl.ca \\ Serge Lefebvre \\ Power Systems and Mathematics \\ Research Institute of Hydro-Quebec \\ Montreal, Quebec, Canada \\ sergelefebvrefcpq@ireq.ca
}

\author{
Maarouf Saad \\ Electrical Engineering Department \\ École de technologie supérieure \\ Montreal, Quebec, Canada \\ Maarouf.saad@etsmtl.ca \\ Dalal Asber \\ Power Systems and Mathematics \\ Research Institute of Hydro-Quebec \\ Montreal, Quebec, Canada \\ Asber.Dalal@ireq.ca
}

\author{
Hasan Mehrjerdi \\ Electrical Engineering Department \\ Qatar University \\ Doha, Qatar \\ Hasan.Mehrjerdi@qu.edu.qa
}

\begin{abstract}
As distribution networks continue in integrating Distributed Generation (DG) units, voltage stability problem becomes an important issue. On other hand, future distribution networks have the ability to support the transmission network. Thus, this work presents an online centralized controller for voltage stability improvement and High-Voltage (HV) side support. The method uses multi-step optimization method to obtain the changes in power injection by DG units while satisfy the system security constrains. The control method follows a security purpose and it is formulated based on the sensitivity of the load and the equivalent impedances to power injections from DG units. An $11 \mathrm{kV}, 77$-bus test network with various DG units is used for this work. Simulation results validate the accuracy of the proposed approach in improving the voltage stability of distribution networks and providing ancillary services to $\mathrm{HV}$ side.
\end{abstract}

Keywords-Distribution Networks; Voltage stability; Smart grids. Voltage control, distributed generation, $\mathrm{HV}$ side support.

\section{INTRODUCTION}

Various approaches have been proposed for voltage control in distribution systems in the presence of DG units. A summary of these methods is available in [1]. Some of control methods are based on optimization programming problems to find the output powers by DG units [2]-[5].

The method proposed in [2] for voltage regulation aims to minimize both the voltage deviation at pilot bus and the power generated by DG units. An approach based on fuzzy and particle swarm optimization methods is proposed in [3] to solve the voltage rise problem and reduce the total losses in the grid in the presence of DG units. The method developed in [4] aims to maximize the power produced by DG units and reduce the total losses. A daily voltage control method is proposed in [5] to reduce the power losses in active distribution systems.

However, the voltage control approaches generally formulate the problem to follow economic purposes. To consider the voltage stability issues, our previous work in [6] formulates the problem to follow a security purpose. It is well known that good voltage magnitudes cannot ensure that the network is stable [7]. The voltage collapse may occur even if the voltages are within acceptable limits [8].

There are several methods are used for voltage stability assessment in distribution systems [9]-[11]. In [9], the assessment method is based on the continuation power flow. In [10], the authors have used a probability theory for stability assessment. In [11], the assessment method is based on a modal analysis. However, they are not suitable for online applications. The approach presented in [6] is fast and can detect the nonlinear characteristics of power systems. The work in [6] also presents a sensitivity analysis on the voltage stability margin of power system to provide guiding information about the control decisions.

Another issue is that high production from DG units with low power consumptions could cause a reverse power flowing to transmission networks. This will definitely cause an overvoltage at some buses of transmission network. According to the European Network Code on Demand Connection (DCC), distribution networks should have the ability to regulate the reactive power flow at transmission/distribution interface. Thus, involving this issue in the voltage (or voltage stability) control of distribution networks is a necessary matter.

In this work, real-time control for DG units is presented to support the transmission network while improving the voltage stability in distribution networks. The work is an extension for the work presented in [6]. The control is based on an optimization programming problem with an objective function to maximize the voltage stability margin to obtain the changes in the active and reactive output powers by DG units. The problem formulation depends on the sensitivity of the load and the equivalent impedances to power injections from DG units. Equability and inequality constraints for 
distribution networks and at transmission/distribution interface are considered.

The remainder of this work is as follows: Section II presents the voltage stability and the impedance-based sensitivity analysis in distribution networks. Section III presents the problem formulation for control the distributed generation for HV support. The results and discussions are presented in section IV while the conclusions are presented in section $\mathrm{V}$.

\section{Voltage StABILITY AND IMPEDANCE-BASED SENSITIVITY ANALYSIS}

According to the Thevenin concept, as the load impedance of a node equals the equivalent impedance of the grid, the maximum power transfer to that load occurs. This means that an indicator $\Psi$ can be defined for voltage stability assessment as:

$$
\Psi=\left|Z_{L}\right|-\left|Z_{e q}\right|
$$

Where $Z_{L}$ is the load impedance. $Z_{e q}$ is the network equivalent impedance. The value of $\Psi$ varies between 1 and zero. If the indicator $\Psi=0$, then the voltage collapse occurs.

The load impedance can be found by taking the ratio between the node voltage and node current. To find the equivalent impedance, the concept of Coupled Single-Port Circuit is used. With the evolution of the measurement devices, this concept can be easily performed to design a model for real-time voltage stability assessment.

The nodal currents of a power system can be expressed as:

$$
\left[\begin{array}{c}
I_{G} \\
-I_{L} \\
0
\end{array}\right]=\left[\begin{array}{ccc}
Y_{G G} & Y_{G L} & Y_{G T} \\
Y_{L G} & Y_{L L} & Y_{L T} \\
Y_{T G} & Y_{T L} & Y_{T T}
\end{array}\right]\left[\begin{array}{c}
V_{G} \\
V_{L} \\
V_{T}
\end{array}\right]
$$

Where $I_{G}$ and $V_{G}$ are current and voltage vector of generator nodes, respectively. $I_{L}$ and $V_{L}$ are current and voltage vector of load nodes, respectively. $V_{T}$ is voltage vector of tie nodes. The terms $Y_{G G}, Y_{G L}, Y_{G T}, Y_{L G}, Y_{L L}, Y_{L T}, Y_{T G}$, $Y_{T L}, Y_{T T}$ are submatrices of the network admittance matrix $Y$.

By analyzing (2) by the same way presented in [6], we obtain that:

$$
Z_{e q, i}=Z_{i i}+\sum_{\substack{\mathrm{j} \epsilon L \\ i \neq j}} Z_{i j} \frac{I_{j}}{I_{i}}
$$

Where $\mathrm{i}$ and $\mathrm{j}$ denote nodes. $Z_{i j}$ is the ij element of load impedance matrix $\left(Y_{L L}^{-1}\right)$ after eliminating the tie buses.

The impedance-based sensitivity analysis method proposed in [6] will be used to achieve the purpose of this work. This method can be summarized by looking to Fig.1. The change in power injection of network buses will cause changes in the load and the equivalent impedances of system buses. For node 4, the system can be reduced to a Thevenin circuit as presented in Fig.1a. It is clear that the change in power injection will cause the load and the equivalent impedances to change by $\Delta Z_{L}, \Delta Z_{e q}$ respectively, as shown in Fig. 1b. This will result in changing the voltage stability margin $\Psi$ of the system.

To obtain the impact of power injection of DG units on the impedances, sensitivity analyses can be performed on the load and the equivalent impedances. According to [6], the impedance sensitivities can be calculated as:

$$
\begin{gathered}
\frac{d Z_{e q, i}}{d u_{x}} \\
=\sum_{\substack{j=1 \\
i \neq j}} \frac{Z_{i j} I_{j}}{I_{i}}\left(\frac{2}{V_{j}^{*}} \frac{d V_{j}}{d u_{x}}-\frac{2}{V_{i}^{*}} \frac{d V_{i}}{d u_{x}}-\frac{1}{V_{j}^{*}} \frac{d V_{j}^{*}}{d u_{x}}+\frac{1}{V_{i}^{*}} \frac{d V_{i}^{*}}{d u_{x}}\right) \\
\frac{d Z_{L, i}}{d u_{x}}=\frac{\left(1-\frac{2 V_{i}}{V_{i}^{*}}\right) \frac{d V_{i}}{d u_{x}}+\frac{V_{i}}{V_{i}^{*}} \frac{d V_{i}^{*}}{d u_{x}}}{I_{i}}
\end{gathered}
$$

Where $u_{x}$ denotes the control variables (i.e. active or reactive power injection by DG units). $\frac{d V_{i}}{d u_{x}}$ and $\frac{d V_{i}{ }^{*}}{d u_{x}}$ are the voltage sensitivities to the control variables and can be calculated using the inverse of Jacobian matrix. Where * denotes conjugate
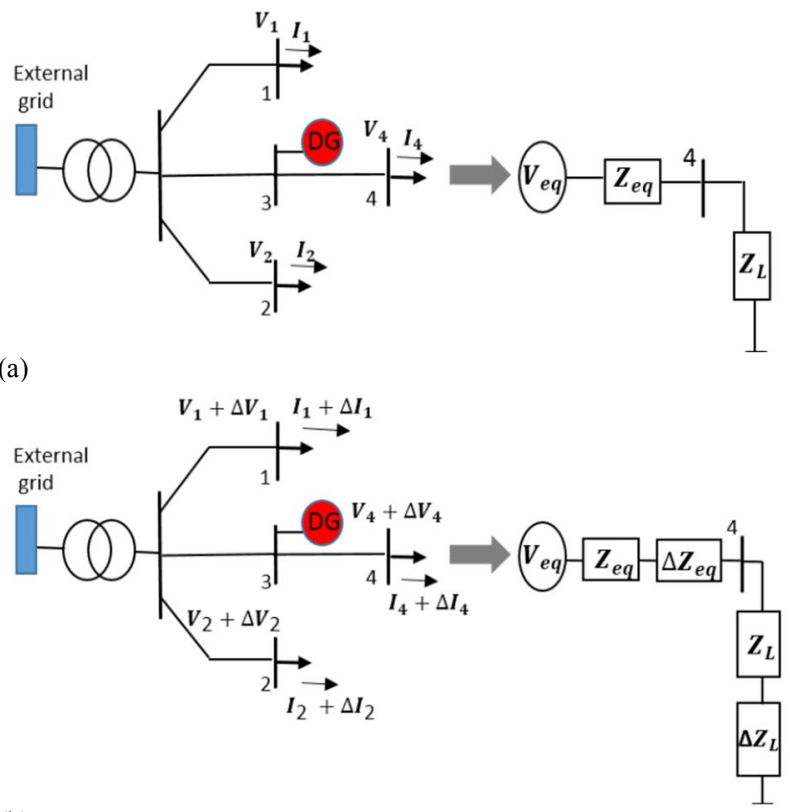

(b)

Fig. 1. A distribution network with an equivalent circuit of node 4 (a) before and (b) after injecting power [6].

\section{PRoblem Formulation OF Distributed GENERATION CONTROL}

An optimization problem based on multi-step actions is used in this work for HV side support. Such multi-step optimization can be found in [6],[12]. The problem formulation is expressed as a Quadratic problem. The performance of the network is predicted over $\mathrm{n}$ steps. The optimization problem has an objective function aiming to maximize the voltage stability margin $(\Psi)$ of the pilot bus. The bus with the minimum margin can be considered as pilot 
bus. Accordingly, this objective function can be formulated as:

$$
\begin{aligned}
\min \sum_{k=0}^{n-1} \| \mid Z_{e q, p} & +\Delta Z_{e q, p}(t+k) \mid \\
& -\left|Z_{L, p}+\Delta Z_{L, p}(t+k)\right| \|^{2}
\end{aligned}
$$

Where $\Delta Z_{L}$ and $\Delta Z_{\text {eq }}$ are the change in the load and the equivalent impedances, respectively. The subletter $\mathrm{p}$ denotes the pilot bus. $t$ represents the time instant.

Equation (6) can be written as:

$$
\begin{array}{r}
\min \sum_{k=0}^{n-1} \| Z_{e q, p}+\sum_{x=1}^{M} \frac{d Z_{e q, p}}{d u_{x}} \Delta u_{x}(t+k) \mid \\
-\left|Z_{L, p}+\sum_{x=1}^{M} \frac{d Z_{L, p}}{d u_{x}} \Delta u_{x}(t+k)\right| \|^{2}
\end{array}
$$

Where $\Delta u_{x}(t+k)$ is the change of the control variable " $\mathrm{x}$ " at time $t+k$.

For economic reasons, it is also important to add another objective function to minimize the change in the power injected by DG units. Such economic reasons are associated with the maintenance and the structural life of control variables. Moreover, it is also necessary to minimize the voltage deviation at network buses, which may be occurred due to the continuous control actions. Thus, the final objective function can be formulated as:

$$
\begin{aligned}
\min \sum_{k=0}^{n-1} \| & \| Z_{e q, p}+\sum_{x=1}^{M} \frac{d Z_{e q, p}}{d u_{x}} \Delta u_{x}(t+k) \mid \\
& -\left|Z_{L, p}+\sum_{x=1}^{M} \frac{d Z_{L, p}}{d u_{x}} \Delta u_{x}(t+k)\right| \|_{G}^{2} \\
& +\sum_{k=0}^{n-1}\|\Delta u(t+k)\|_{G}^{2} \\
& +\sum_{k=0}^{n-1}\|\Delta V(t+k)\|_{G}^{2}+\|\varepsilon\|_{H}^{2}
\end{aligned}
$$

Subject to:

$$
\begin{gathered}
-\varepsilon_{1} A+V_{i}^{\text {min }} \leq V_{i}(t+k) \leq V_{i}^{\max }+\varepsilon_{2} A \\
V_{i}(t+k)=V_{i}(t+k-1)+\frac{\partial|V|}{\partial u} \Delta u_{i}(t+k) \\
\Delta Q_{x}^{\text {min }} \leq \Delta Q_{x}(t+k) \leq \Delta Q_{x}^{\max } \\
\Delta P_{x}^{\text {min }} \leq \Delta P_{x}(t+k) \leq \Delta P_{x}^{\max } \\
P_{x}^{\text {min }} \leq P_{x}(t+k) \leq P_{x}^{\max } \\
Q_{x}^{\text {min }} \leq Q_{x}(t+k) \leq Q_{x}^{\max }
\end{gathered}
$$

Where $\frac{d Z_{L, p}}{d u}=\left[\frac{d Z_{L, p}}{d P}, \frac{d Z_{L, p}}{d Q}\right]$ and $\frac{d Z_{e q, p}}{d u}=\left[\frac{d Z_{e q, p}}{d P}, \frac{d Z_{e q, p}}{d Q}\right]$ represent the sensitivity vectors of the impedances with respect to the control variables. $\Delta u=[\Delta P, \Delta Q]^{T}$ is the change vector of the control variables. ' $\mathrm{T}$ ' represents array transposition. $\Delta V$ is the vector of the deviation in the node voltages. $\varepsilon=\left[\varepsilon_{1}, \varepsilon_{2}\right]^{\mathrm{T}}$ is the slack variable vector used for constraint relaxation. 'A' is a unitary vector. $G$ and $\mathrm{H}$ are weight matrices. $V_{i}(t+k)$ is the predicted voltage. $V_{i}(t+$ $k-1)$ is the measured voltage. $\frac{\partial|V|}{\partial u}=\left[\frac{d|V|}{d P}, \frac{d|v|}{d Q}\right]$ is the sensitivity matrix of bus voltages with respect to the real and reactive power injections. $P$ and $Q$ denote the active and reactive power injection by DG units. "min" and "max" denote minimum and maximum values, respectively.

For HV side support, it is necessary to add a constraint that control the voltage or reactive power at the transmission/ distribution interface. For controlling the external voltage, the following constraint can be used:

$$
\begin{gathered}
-\varepsilon_{3}+V_{e x}^{\text {min }} \leq V_{e x}(t+k) \leq V_{e x}^{\max }+\varepsilon_{4} \\
V_{e x}(t+k)=V_{e x}(t+k-1)+\frac{\partial\left|V_{e x}\right|}{\partial u} \Delta u_{i}(t+k)
\end{gathered}
$$

Where $V_{\text {ex }}$ denotes the external voltage (the interface point between transmission and distribution networks). $\varepsilon_{3}$ and $\varepsilon_{4}$ are slack variables used to relax the external bus voltage.

\section{SiMULATION RESULTS}

To demonstrate the validity of the present method for HV side support, $11 \mathrm{kV}, 75$ distribution system having $22 \mathrm{DG}$ units is used in this work. The system topology is presented in Fig. 2. The system information can be found in [13]. MATLAB and LINGO softwares were used to investigate the results.

This work assumes that each DG unit has a rating of 4 MVA. Each DG unit is not allowed to provide or absorb power more than $0.3 \mathrm{MW}$ (or 0.3 MVAr). This work also assumes that the change cost of the real power or slack values are greater than the change cost of reactive power by 10 and 800 times respectively. The acceptable range for voltages is assumed to be within $[0.98,1.04]$ p.u. For the HV side, it is requested to maintain the voltage at transmission/distribution interface (V1000) within [1.0 and 1.01] p.u. If the reactive power is able to solve the problem, the active power will not be involved in the control.

Fig. 3 shows the voltage at transmission/distribution interface (i.e. V1000). The voltage before $40 \mathrm{sec}$ is violated. It is assumed that the control takes place at $40 \mathrm{sec}$. Since V1000 is less than the minimum voltage limit, distribution network will be required to inject power into bus 1000. By solving the optimization problem, set-points of DG units will be updated to increase their power injections. It is clear that six periods (each of around $10 \mathrm{sec}$ ) are needed to restore V1000 inside the acceptable range. This HV support occurs by sequence of changes of the reactive power injected from 
DG units. The successful control of the external bus voltage demonstrates the validity of the presented control method of DG units.

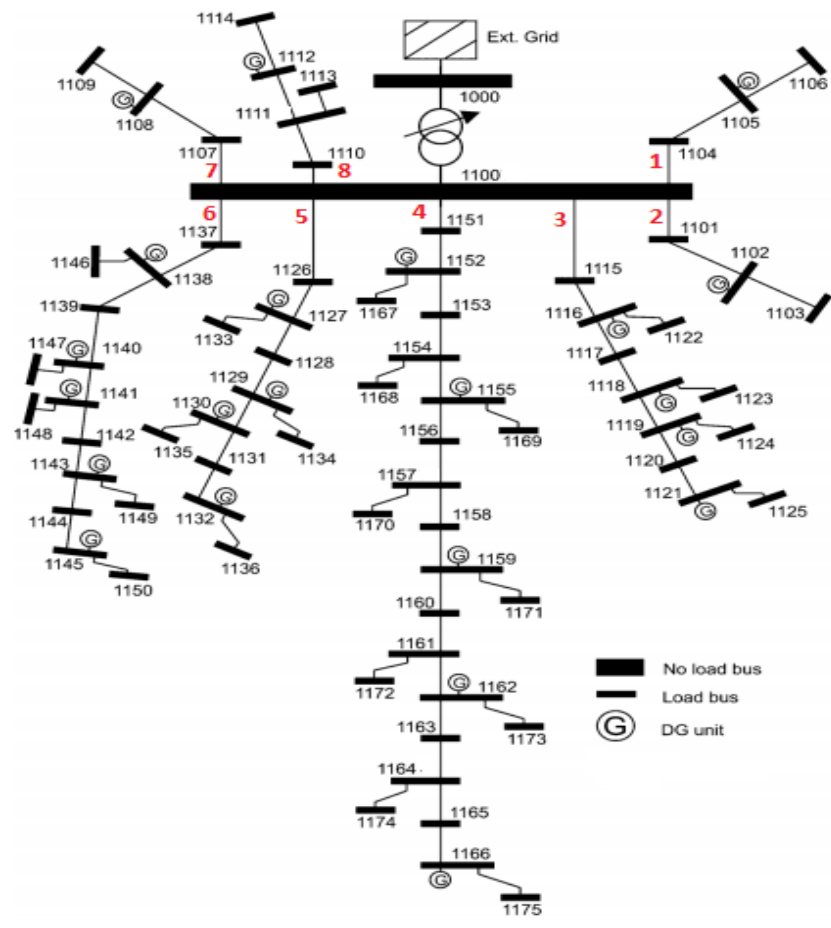

Fig.2: Topology of the distribution network [6]

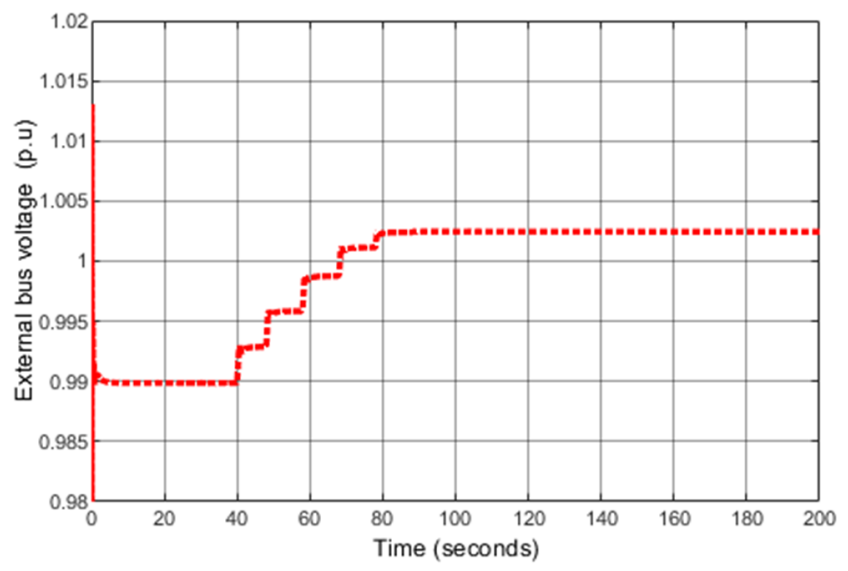

Fig.3. Control the voltage at Transmission/distribution interface

Fig.4 shows the voltage of some buses of distribution networks. It can be seen that some voltages are less than the voltage limits during the period [0 40] sec. At $40 \mathrm{sec}$ the presented method was able to regulate the voltages and restore the violated ones inside the normal limits during HV side support. Moreover, from the analysis, it was clear the voltage stability of the distribution network was improvement by $1.05 \%$ after performing the control actions.

Fig.5 shows the optimal dispatch of some DG units. It is clear that six control actions are triggered by DG units to solve the problem. It is clear that the DG unit located at bus 1105 supplies the largest amount of reactive power. This is due to fact that bus 1105 is the nearest bus to transmission/distribution interface. In contrast, the DG units located far away from the interface point supply less amount of reactive power. This also demonstrates the accuracy of the presented control in supplying the HV side.

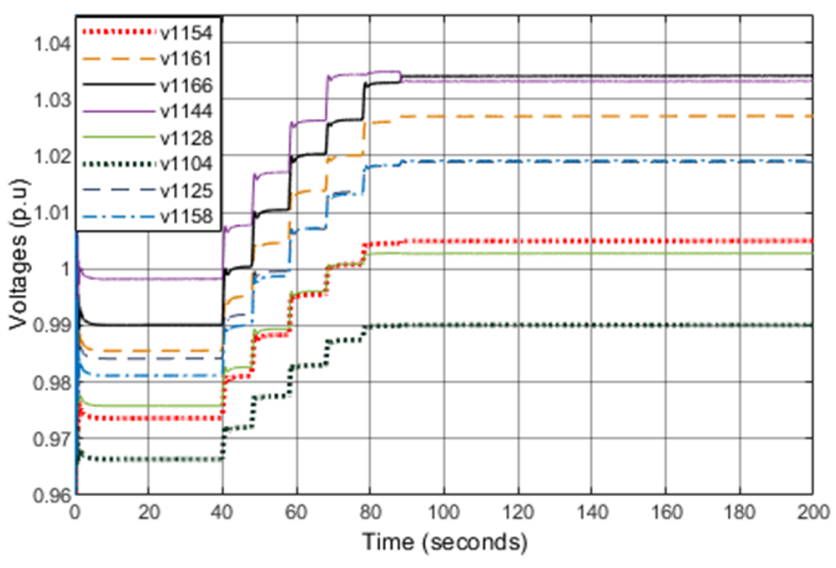

Fig.4. Voltages of some buses

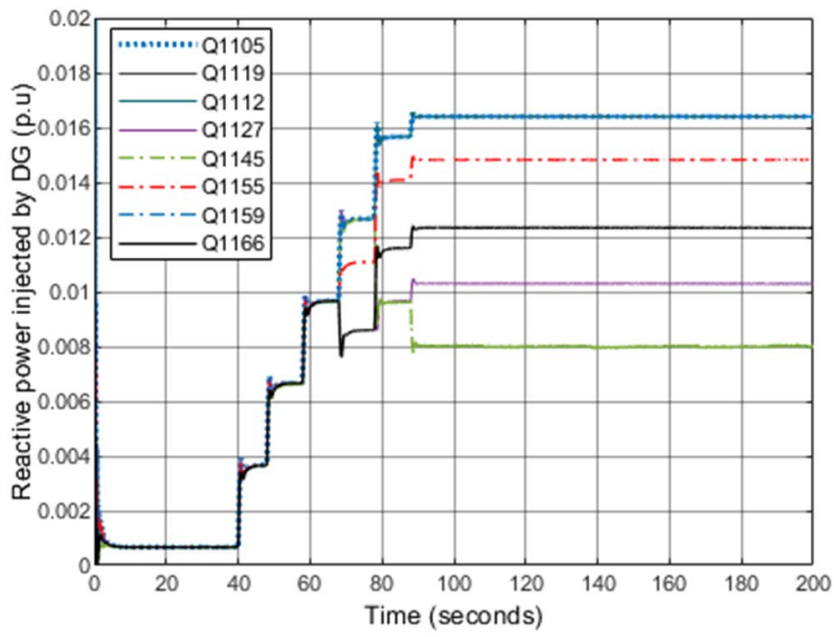

Fig.5. Reactive power injected by some DGs

Regarding the calculation time, less than one sec is required to solve the presented optimization problem. However, $10 \mathrm{sec}$ is selected per each period to a) consider the time required to transmit the measurements and b) wait to avoid the transient measurements.

It is worth mentioning that the reactive power outputs by DG units were able to solve the problem while meeting all the constraints. That why only the reactive powers are presented in the results.

\section{CONCLUSIONS}

In this work, a fast method to control DG units is presented in order to support HV side and improving the voltage stability in distribution networks. The method is based on a multi-step optimization programming problem to obtain the changes in the power injected by DG units.

The method is validated on $11 \mathrm{KV}, 75$ bus distribution network having 22 DG units. The results show that the presented control approach is able to regulate the voltage at the transmission/distribution interface and improving the 
voltage stability of distribution networks. The dispatch of the power injection by DG units also demonstrates the accuracy of the approach in voltage control and HV side support. The dynamic behavior of DG units is also shown through the results.

Our future work is to develop a method for distribution networks to control the voltage stability margin at transmission networks.

\section{ACKNOWLEDGMENT}

This work was supported by the National Priorities Research Program under Grant 11S-1125-170027 from the Qatar National Research Fund (a member of Qatar Foundation).

\section{REFERENCES}

[1] V. A. Evangelopoulos, P. S. Georgilakis, and N. D. Hatziargyriou, Optimal operation of smart distribution networks: A review of models, methods and future research, Electric Power Systems Research, 140 (2016) 95-106

[2] Castro, J. R., Saad, M., Lefebvre, S., Asber, D., \& Lenoir, L., Optimal voltage control in distribution network in the presence of DGs, International Journal of Electrical Power \& Energy Systems, 78 (2016) 239-247.

[3] [10] H.T. Yang, and J.T. Liao. , MF-APSO-based multiobjective optimization for PV system reactive power regulation, IEEE Transactions on Sustainable Energy, 6 (4) (2015) 1346-1355.

[4] D. Jakus, J. Vasilj, and P. Sarajcev, Voltage control in MV distribution networks through coordinated control of tap changers and renewable energy sources, In PowerTech, 2015 IEEE Eindhoven, IEEE, (2015) 1-

[5] Degefa, M. Z., Lehtonen, M., Millar, R. J., Alahäivälä, A., \& Saarijärvi, E. , Optimal voltage control strategies for day-ahead active distribution network operation, Electric Power Systems Research, 127 (2015) 4152 .

[6] Alzaareer, K., Saad, M., Asber, D., Lefebvre, S., \& Lenoir, L. (2020). Impedance sensitivity-based corrective method for online voltage control in smart distribution grids. Electric Power Systems Research, $181,106188$.

[7] P. A. Lof, T. Smed, G. Andersson, and D. J. Hill, Fast calculation of a voltage stability index, IEEE Trans. Power Systems, 7(1) (1992) 5464.

[8] P. Kundur, Power System Stability and Control. McGraw-Hill Professional, 1994.

[9] X. Dou, S. Zhang, L. Chang, Z. Wu, W. Gu, M. Hu, \& X. Yuan., An improved CPF for static stability analysis of distribution systems with high DG penetration, International Journal of Electrical Power \& Energy Systems, 86 (2017) 177-188.

[10] K.Y, Liu, W. Sheng, L.Hu, Y. Liu, X. Meng, and D. Jia, Simplified probabilistic voltage stability evaluation considering variable renewable distributed generation in distribution systems, IET Generation, Transmission \& Distribution, 9(12) (2015) 1464-1473.

[11] H. M. Chou, and K. L. Butler-Purry, Investigation of Voltage Stability in Three-Phase Unbalanced Distribution Systems with DG using Modal Analysis Technique, In North American Power Symposium (NAPS), (2014) 1-6.

[12] Valverde, Gustavo, and Thierry Van Cutsem. "Model predictive control of voltages in active distribution networks." IEEE Transactions on Smart Grid 4, no. 4 (2013): 2152-2161.

[13] United Kingdom Generic Distribution Network (UKGDS). [Online]. Available: http://sedg.ac.uk 